\title{
Symmetric Textures in SO(10) and LMA Solution for Solar Neutrinos
}

\author{
Mu-Chun Chen* and K.T. Mahanthappa ${ }^{\dagger}$ \\ ${ }^{*}$ HET Group, Physics Department, Brookhaven National Laboratory, Upton, NY 11973 \\ ${ }^{\dagger}$ Department of Physics, University of Colorado, Boulder, CO 80309
}

\begin{abstract}
A model based on SUSY SO(10) combined with SU(2) family symmetry is constructed. In contrast with the commonly used effective operator approach, 126-dimensional Higgs fields are utilized to construct the Yukawa sector. R-parity symmetry is thus preserved at low energies. The symmetric mass textures arising from the left-right symmetry breaking chain of $\mathrm{SO}(10)$ give rise to very good predictions for quark and lepton masses and mixings. The prediction for $\sin 2 \beta$ agrees with the average of current bounds from $\mathrm{BaBar}$ and Belle. In the neutrino sector, our predictions are in good agreement with results from atmospheric neutrino experiments. Our model accommodates the LMA solution to the solar neutrino anomaly. The prediction of our model for the $\left|U_{e v_{3}}\right|$ element in the MNS matrix is close to the sensitivity of current experiments; thus the validity of our model can be tested in the near future. We also investigate the correlation between the $\left|U_{e v_{3}}\right|$ element and $\tan ^{2} \theta_{\odot}$ in a general two-zero neutrino mass texture.
\end{abstract}

$\mathrm{SO}(10)$ has long been thought to be an attractive candidate for a grand unified theory (GUT) for a number of reasons: First of all, it unifies all the 15 known fermions with the right-handed neutrino for each family into one 16-dimensional spinor representation. The seesaw mechanism then arises very naturally, and the non-zero neutrino masses can thus be explained. Since a complete quark-lepton symmetry is achieved, it has the promise for explaining the pattern of fermion masses and mixing. Because B-L contained in $\mathrm{SO}(10)$ is broken in symmetry breaking chain to the SM, it also has the promise for baryogenesis. Recent atmospheric neutrino oscillation data from SuperKamiokande indicates non-zero neutrino masses. This in turn gives very strong support to the viability of $\mathrm{SO}(10)$ as a GUT group. Models based on $\mathrm{SO}(10)$ combined with discrete or continuous family symmetry have been constructed to understand the flavor problem. Most of the models utilize "lopsided" mass textures which usually require more parameters and therefore are less constrained. Furthermore, the right-handed neutrino Majorana mass operators in most of these models are made out of $16_{H} \times 16_{H}$ which breaks the R-parity at a very high scale. The aim of this talk, based on Ref.[1, 2, 3], is to present a realistic model based on supersymmetric $S O(10)$ combined with $S U(2)$ family symmetry which successfully predicts the low energy fermion masses and mixings. Since we utilize symmetric mass textures and $\overline{126}$-dimensional Higgs representations for the right-handed neutrino Majorana mass operator, our model is more constrained in addition to having R-parity conserved. We first discuss the viable phenomenology of mass textures followed by the model which accounts for it, and then the implications of the model for neutrino mixing, $\mathrm{CP}$ violation, and neutrinoless double beta decay are 
presented.

The set of up- and down-quark mass matrix combination is given by, at the GUT scale,

$$
M_{u}=\left(\begin{array}{ccc}
0 & 0 & a e^{i \gamma_{a}} \\
0 & b e^{i \gamma_{b}} & c e^{i \gamma_{c}} \\
a e^{i \gamma_{a}} & c e^{i \gamma_{c}} & e^{i \gamma_{d}}
\end{array}\right) d v_{u}, \quad M_{d}=\left(\begin{array}{ccc}
0 & e e^{i \gamma_{e}} & 0 \\
e e^{i \gamma_{e}} & f e^{i \gamma_{f}} & 0 \\
0 & 0 & e^{i \gamma_{h}}
\end{array}\right) h v_{d}
$$

with $a \simeq b \ll c \ll 1$, and $e \ll f \ll 1$. Symmetric mass textures arise naturally if $\mathrm{SO}(10)$ breaks down to the SM through the left-right symmetric breaking chain $S U(4) \times S U(2)_{L} \times S U(2)_{R}$. $\mathrm{SO}(10)$ relates the up-quark mass matrix to the Dirac neutrino mass matrix, and the down-quark mass matrix to the charged lepton mass matrix. To achieve the GeorgiJarlskog relations, a factor of -3 is needed in the $(2,2)$ entry of the charged lepton mass matrix,

$$
M_{e}=\left(\begin{array}{ccc}
0 & e e^{i \gamma_{e}} & 0 \\
e e^{i \gamma_{e}} & -3 f e^{i \gamma_{f}} & 0 \\
0 & 0 & e^{i \gamma_{h}}
\end{array}\right) h v_{d}
$$

This factor of -3 can be accounted for by the $\mathrm{SO}(10) \mathrm{CG}$ coefficients associated with $\overline{126}-$ dimensional Higgs representations. The smallness of the neutrino masses is explained by the type I seesaw mechanism. The Dirac neutrino mass matrix is identical to the mass matrix of the up-quarks in the framework of $\mathrm{SO}(10)$

$$
M_{v_{L R}}=\left(\begin{array}{ccc}
0 & 0 & a e^{i \gamma_{a}} \\
0 & b e^{i \gamma_{b}} & c e^{i \gamma_{c}} \\
a e^{i \gamma_{a}} & c e^{i \gamma_{c}} & e^{i \gamma_{d}}
\end{array}\right) d v_{u}
$$

The right-handed neutrino sector is an unknown sector. It is only constrained by the requirement that it gives rise to a bi-maximal mixing pattern and a hierarchical mass spectrum at low energies. To achieve this, we consider an effective neutrino mass matrix of the form

$$
M_{v_{L L}}=M_{v_{L R}}^{T} M_{v_{R R}}^{-1} M_{v_{L R}}=\left(\begin{array}{ccc}
0 & 0 & t \\
0 & 1 & 1+t^{n} \\
t & 1+t^{n} & 1
\end{array}\right) \frac{d^{2} v_{u}^{2}}{M_{R}}
$$

The effective neutrino mass matrix of this form is obtained if the right-handed neutrino mass matrix has the same texture as that of the Dirac neutrino mass matrix,

$$
M_{v_{R R}}=\left(\begin{array}{ccc}
0 & 0 & \delta_{1} \\
0 & \delta_{2} & \delta_{3} \\
\delta_{1} & \delta_{3} & 1
\end{array}\right) M_{R}
$$

and if the elements $\delta_{i}$ are of the right orders of magnitudes, determined by $\delta_{i}=f_{i}(a, b, c, t, \theta)$, where $\theta \equiv\left(\gamma_{b}-2 \gamma_{c}-\gamma_{d}\right)$. Note that $M_{v_{L L}}$ has the same texture as that of $M_{v_{L R}}$ and $M_{v_{R R}}$, thus the seesaw mechanism is form invariant. A generic feature of mass matrices of the type given in Eq.(4) is that they give rise to bi-maximal mixing pattern. After diagonalizing this mass matrix, one can see immediately that the squared mass difference between $m_{v_{1}}^{2}$ and $m_{v_{2}}^{2}$ is of the order of $O\left(t^{3}\right)$, while the squared mass difference between $m_{v_{2}}^{2}$ and $m_{v_{3}}^{2}$ 
is of the order of $O(1)$, in units of $\Lambda$. For $t \ll 1$, the phenomenologically favored relation $\Delta m_{a t m}^{2} \gg \Delta m_{\odot}^{2}$ is thus obtained.

The SU(2) family symmetry is implemented á la the Froggatt-Nielsen mechanism. The heaviness of the top quark and to suppress the SUSY FCNC together suggest that the third family of matter fields transform as a singlet and the lighter two families of matter fields transform as a doublet under SU(2). In the family symmetric limit, only the third family has non-vanishing Yukawa couplings. SU(2) breaks down in two steps: $S U(2) \stackrel{\varepsilon M}{\longrightarrow} U(1) \stackrel{\varepsilon^{\prime} M}{\longrightarrow}$ nothing, where $\varepsilon^{\prime} \ll \varepsilon \ll 1$ and $M$ is the family symmetry scale. These small parameters $\varepsilon$ and $\varepsilon^{\prime}$ are the ratios of the vacuum expectation values of the flavon fields to the family symmetry scale. A discrete symmetry $\left(Z_{2}\right)^{3}$ is needed to avoid unwanted couplings. The field content of our model is then given by

- matter fields

$$
\psi_{a} \sim(16,2)^{-++} \quad(a=1,2), \quad \psi_{3} \sim(16,1)^{+++}
$$

- Higgs fields:

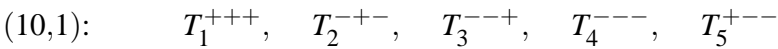

$$
\begin{aligned}
& (\overline{126}, 1): \quad \bar{C}^{---}, \quad \bar{C}_{1}^{+++}, \quad \bar{C}_{2}^{++-}
\end{aligned}
$$

- Flavon fields:

$$
\begin{array}{llll}
(1,2): & \phi_{(1)}^{++-}, & \phi_{(2)}^{+-+}, & \Phi^{-+-} \\
(1,3): & S_{(1)}^{+--}, & S_{(2)}^{---}, & \Sigma^{++-}
\end{array}
$$

and the superpotential of our model which generates fermion masses is given by

$$
\begin{aligned}
& W=W_{D(\text { irac })}+W_{M(\text { ajorana })} \\
& W_{D}=\psi_{3} \psi_{3} T_{1}+\frac{1}{M} \psi_{3} \psi_{a}\left(T_{2} \phi_{(1)}+T_{3} \phi_{(2)}\right)+\frac{1}{M} \psi_{a} \psi_{b}\left(T_{4}+\bar{C}\right) S_{(2)}+\frac{1}{M} \psi_{a} \psi_{b} T_{5} S_{(1)} \\
& W_{M}=\psi_{3} \psi_{3} \bar{C}_{1}+\frac{1}{M} \psi_{3} \psi_{a} \Phi \bar{C}_{2}+\frac{1}{M} \psi_{a} \psi_{b} \Sigma \bar{C}_{2}
\end{aligned}
$$

where $T_{i}$ 's and $\bar{C}_{i}$ 's are the 10 and $\overline{126}$ dimensional Higgs representations of $\mathrm{SO}(10)$ respectively, and $\Phi$ and $\Sigma$ are the doublet and triplet of $S U(2)$, respectively. Detailed quantum number assignment and the VEVs acquired by various scalar fields are given in Ref.[1]. This superpotential gives rise to the mass textures given in Eq.(11)-(5):

$$
\begin{gathered}
M_{u, v_{L R}}=\left(\begin{array}{ccc}
0 & 0 & \left\langle 10_{2}^{+}\right\rangle \varepsilon^{\prime} \\
0 & \left\langle 10_{4}^{+}\right\rangle \varepsilon & \left\langle 10_{3}^{+}\right\rangle \varepsilon \\
\left\langle 10_{2}^{+}\right\rangle \varepsilon^{\prime} & \left\langle 10_{3}^{+}\right\rangle \varepsilon & \left\langle 10_{1}^{+}\right\rangle
\end{array}\right)=\left(\begin{array}{ccc}
0 & 0 & r_{2} \varepsilon^{\prime} \\
0 & r_{4} \varepsilon & \varepsilon \\
r_{2} \varepsilon^{\prime} & \varepsilon & 1
\end{array}\right) M_{U} \\
M_{d, e}=\left(\begin{array}{ccc}
0 & \left\langle 10_{5}^{-}\right\rangle \varepsilon^{\prime} & 0 \\
\left\langle 10_{5}^{-}\right\rangle \varepsilon^{\prime} & (1,-3)\langle\overline{126}\rangle \varepsilon & 0 \\
0 & 0 & \left\langle 10_{1}^{-}\right\rangle
\end{array}\right)=\left(\begin{array}{ccc}
0 & \varepsilon^{\prime} & 0 \\
\varepsilon^{\prime} & (1,-3) p \varepsilon & 0 \\
0 & 0 & 1
\end{array}\right) M_{D}
\end{gathered}
$$

where $M_{U} \equiv\left\langle 10_{1}^{+}\right\rangle, M_{D} \equiv\left\langle 10_{1}^{-}\right\rangle, r_{2} \equiv\left\langle 10_{2}^{+}\right\rangle /\left\langle 10_{1}^{+}\right\rangle, r_{4} \equiv\left\langle 10_{4}^{+}\right\rangle /\left\langle 10_{1}^{+}\right\rangle$and $p \equiv\left\langle\overline{126}^{-}\right\rangle /\left\langle 10_{1}^{-}\right\rangle$. The righthanded neutrino mass matrix is

$$
M_{v_{R R}}=\left(\begin{array}{ccc}
0 & 0 & \left\langle\overline{126}_{2}^{0}\right\rangle \delta_{1} \\
0 & \left\langle\overline{126}_{2}^{\prime}\right\rangle^{0} & \left\langle\delta_{2}{ }^{\prime}{ }_{2}\right\rangle \delta_{3} \\
\left\langle\overline{126}_{2}^{0}\right\rangle \delta_{1} & \left\langle\overline{126}_{2}^{\prime}\right\rangle\left\langle\delta_{3}\right. & \left\langle\overline{126}_{1}^{\prime}\right\rangle
\end{array}\right)=\left(\begin{array}{ccc}
0 & 0 & \delta_{1} \\
0 & \delta_{2} & \delta_{3} \\
\delta_{1} & \delta_{3} & 1
\end{array}\right) M_{R}
$$




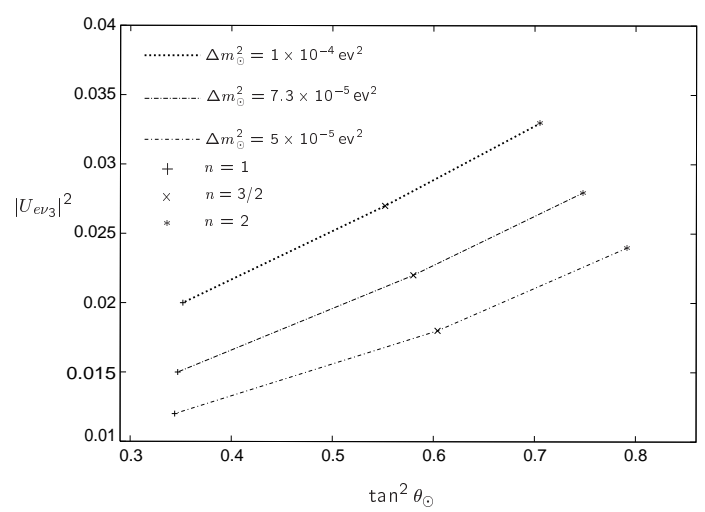

FIGURE 1. Correlation between $\left|U_{e v_{3}}\right|^{2}$ and $\tan ^{2} \theta_{\odot} . \Delta m_{a t m}^{2}$ is $2.8 \times 10^{-3} \mathrm{eV}^{3}$. The dotted line corresponds to the upper bound $\Delta m_{\odot}^{2}=10^{-4} \mathrm{eV}^{2}$; the dotted-long-dashed line corresponds to the best fit value $\Delta m_{\odot}^{2}=7.3 \times 10^{-5} \mathrm{eV}^{2}$; the dotted-short-dashed line corresponds to the lower bound $\Delta m_{\odot}^{2}=5 \times 10^{-5} \mathrm{eV}^{2}$.

with $M_{R} \equiv\left\langle\overline{126}_{1}^{\prime}\right\rangle$. Note that, since we use $\overline{126}$-dimensional representations of Higgses to generate the heavy Majorana neutrino mass terms, R-parity is preserved at all energies.

With values of $m_{f},(f=u, c, t, e, \mu, \tau)$ and those of $\left|V_{u s, u b, c b}\right|$ at the weak scale, the input parameters at the GUT scale are determined. The predictions for the charged fermion masses and CKM mixing of our model at $M_{Z}$ which are summarized below including 2-loop RGE effects are in good agreements with the experimental values:

$$
\begin{array}{lllll}
\frac{m_{s}}{m_{d}}=25, & m_{s}=85.66 \mathrm{MeV}, & m_{b}=3.147 \mathrm{GeV}, & \left|V_{u d}\right|=0.9751, & \left|V_{c d}\right|=0.2218 \\
\left|V_{c s}\right|=0.9744, & \left|V_{t d}\right|=0.005358, & \left|V_{t s}\right|=0.03611, & \left|V_{t b}\right|=0.9993, & J_{C P}^{q}=1.748 \times 10^{-5} \\
\sin 2 \alpha=-0.8913, & \sin 2 \beta=0.7416, & \gamma=34.55^{0} . & &
\end{array}
$$

Using the mass square differences $\Delta m_{a t m}^{2}=2.78 \times 10^{-3} \mathrm{eV}^{2}$ and $\Delta m_{\odot}^{2}=7.25 \times 10^{-5} \mathrm{eV}^{2}$ for the LOW solution as input parameters, we determine $\left(t, M_{R}\right)=\left(0.35,5.94 \times 10^{12} \mathrm{GeV}\right)$, and correspondingly $\left(\delta_{1}, \delta_{2}, \delta_{3}\right)=\left(0.00119,0.000841 e^{i(0.220)}, 0.0211 e^{-i(0.029)}\right)$. The three mass eigenvalues are predicted to be $\left(m_{v_{1}}, m_{v_{2}}, m_{v_{3}}\right)=(0.00363,0.00926,0.0535) \mathrm{eV}$, and the mixing angles are predicted to be $\sin ^{2} 2 \theta_{\text {atm }}=1, \tan ^{2} \theta_{\odot}=0.58, \sin ^{2} \theta_{13}=0.022$. These predictions agree with current bounds from experiments within $1 \sigma$. The strengths of $\mathrm{CP}$ violation in the lepton sector are $\left(J_{C P}^{l}, \alpha_{31}, \alpha_{21}\right)=(-0.00690,0.490,-2.29)$, and the matrix element for the neutrinoless double $\beta$ decay is given by $|<m>|=2.22 \times 10^{-3} \mathrm{eV}$. The correlation between $\left|U_{e v_{3}}\right|^{2}$ and $\tan ^{2} \theta_{\odot}$ is plotted in Fig. 1. A comparison of the predictions for $\sin ^{2} 2 \theta_{13}$ and $(\alpha, \beta, \gamma)$ from different $\mathrm{SO}(10)$ models is given in Ref.[4]. This work was supported by US DOE under Grant No. DEAC02-98CH10886 and DE-FG03-95ER40894.

\section{REFERENCES}

1. M. -C. Chen and K. T. Mahanthappa, Phys. Rev. D62, 113007 (2000).

2. M. -C. Chen and K. T. Mahanthappa, Phys. Rev. D65, 053010 (2002).

3. M. -C. Chen and K. T. Mahanthappa, Phys. Rev. D68, 017301 (2003).

4. M. -C. Chen and K. T. Mahanthappa, arXiv:hep-ph/0305088. 